\title{
Editorial
}

\section{Plasma Medicine Technologies}

\author{
Nagendra Kumar Kaushik ${ }^{1, *}\left(\mathbb{D}\right.$, Sander Bekeschus ${ }^{2}\left(\mathbb{D}\right.$, Hiromasa Tanaka ${ }^{3}\left(\mathbb{D}\right.$, Abraham Lin $^{4}(\mathbb{D}$ \\ and Eun Ha Choi ${ }^{1, *(D)}$
}

1 Plasma Bioscience Research Center, Applied Plasma Medicine Center, Department of Electrical and Biological Physics, Kwangwoon University, Seoul 01897, Korea

2 ZIK Plasmatis, Leibniz Institute for Plasma Science and Technology, 17489 Greifswald, Germany; sander.bekeschus@gmail.com

3 Center for Low-Temperature Plasma Sciences, Nagoya University, Nagoya 464-8601, Japan; htanaka@plasma.engg.nagoya-u.ac.jp

4 Center for Oncological Research, Research Group PLASMANT, University of Antwerp, Universiteitsplein 1, BE-2610 Wilrijk-Antwerp, Belgium; abraham.lin@uantwerpen.be

* Correspondence: kaushik.nagendra@kw.ac.kr (N.K.K.); ehchoi@kw.ac.kr (E.H.C.)

Citation: Kaushik, N.K.; Bekeschus,

S.; Tanaka, H.; Lin, A.; Choi, E.H.

Plasma Medicine Technologies. Appl. Sci. 2021, 11, 4584. https://doi.org/ 10.3390/app11104584

Received: 28 April 2021

Accepted: 13 May 2021

Published: 18 May 2021

Publisher's Note: MDPI stays neutral with regard to jurisdictional claims in published maps and institutional affiliations.

Copyright: (c) 2021 by the authors. Licensee MDPI, Basel, Switzerland. This article is an open access article distributed under the terms and conditions of the Creative Commons Attribution (CC BY) license (https:// creativecommons.org/licenses/by/ $4.0 /)$.

\begin{abstract}
This Special Issue, entitled "Plasma Medicine Technologies", covers the latest remarkable developments in the field of plasma bioscience and medicine. Plasma medicine is an interdisciplinary field that combines the principles of plasma physics, material science, bioscience, and medicine, towards the development of therapeutic strategies. A study on plasma medicine has yielded the development of new treatment opportunities in medical and dental sciences. An important aspect of this issue is the presentation of research underlying new therapeutic methods that are useful in medicine, dentistry, sterilization, and, in the current scenario, that challenge perspectives in biomedical sciences. This issue is focused on basic research on the characterization of the bioplasma sources applicable to living cells, especially to the human body, and fundamental research on the mutual interactions between bioplasma and organic-inorganic liquids, and bio or nanomaterials.
\end{abstract}

Keywords: nonthermal plasma; plasma medicine; cancer; dental material; stem cells; neuronal regeneration; in vivo; ex vivo; bio-sterilization; decontamination

\section{Introduction}

This Special Issue provides an applied and basic forum for exchanging innovative research ideas or experimental performances that link the latest plasma medicine research in cancer biology, dental material development, stem cell differentiation, neuronal regeneration, water decontamination, and sterilization applications, including in vivo and ex vivo studies. This issue encompasses analytical and empirical investigations; comprehensive reviews of relevant biological research; and theoretical frameworks and case studies of valuable applications in this plasma medicine area. Several papers were submitted to this Special Issue, of which 10 high-quality research papers were accepted. When looking back at this Special Issue, various topics have been addressed, mainly plasma-based cancer treatments (four articles), dental application (one article), stem cell and neuronal differentiation (two articles), immune activation (one article), microbial treatment (two articles), and environmental application (two articles). We are thankful to all these authors, and it is our great pleasure to create a compilation of high-quality articles in this issue. The following subsections provide a summary of each of the papers published.

\section{Contributions \\ 2.1. Cancer Treatment Application}

Cancer remains one of the biggest challenges to clinical and biological research because it is the leading cause of death worldwide. Conventional treatment procedures can cause many adverse effects and cause resistance toward therapeutics. Thus, alternative 
approaches to control and treat cancer are gaining more attention. Mitra et al. have demonstrated that extracts derived from plasma-treated mushrooms induce the death of breast cancer cells [1]. They also showed that plasma-treated mushroom groups produced high levels of phenolic and flavonoid compounds. They postulate that exposure to plasma can enhance the active properties and yield of mushroom constituents that could be useful for suppressing cancer cell growth.

Adhikari et al. investigated the role of a plasma-activated medium (PAM) on a human melanoma cancer cells' xenograft [2]. They performed gene expression analysis, a membrane degradation study, and a melanoma biomarker estimation using blood serum from xenograft mice bearing melanoma cells. They found that a PAM contains ROS, which induces the intracellular ROS level and activates the P53-signalling cascade, which consequently causes cancer cell death by activating apoptotic gene expression and degrading macromolecules. In another article, “Ex Vivo Exposure of Human Melanoma Tissue to Cold Physical Plasma Elicits Apoptosis and Modulates Inflammation", the authors used primary malignant melanoma metastasis tissues from a stage IV patient's ex vivo to analyze the susceptibility of this human melanoma tissue to plasma-induced cell death [3]. Quantitative imaging was used to analyze the amount of apoptotic cells in an unbiased fashion. Strikingly, it was found that the plasma treatment significantly increased the number of apoptotic cells compared to the untreated control tissue. This supports the motivation for using this novel technology in a clinical setting. Along those lines, it was also observed that the secretory profiles of the plasma-treated tissue changed, suggesting that the tumor-toxic plasma treatment also modulated inflammation inside the human melanoma tissue. Skin cancer was also dealt with in a review of Julia Berner and colleagues' "Medical Gas Plasma Treatment in Head and Neck Cancer-Challenges and Opportunities" [4]. In this work, the current state-of-the-art knowledge on the plasma treatment of head and neck cancer is summarized for in vitro and in vivo studies. The team of authors concluded that plasma treatment not only might be a suitable treatment modality for these often-superficial tumor lesions but also proves to be very acceptable from the safety point of view, as no severe side effects have so far been described in head and neck cancer patients exposed to plasma.

\subsection{Dental Application}

Recently, several investigations have attempted to enhance the bond strength among core resin and epoxy resin-based fiber posts. Kim et al. showed that plasma treatment can be used to increase the bond strength between an epoxy resin-based fiber post and core resin [5]. Plasma treatment improved the bond strength between core resin and an epoxy resin-based fiber post by altering its surface chemically. The outcomes were comparable to a standard combined exposure to $4 \%$ hydrofluoric acid and silane, but better than an exposure to $4 \%$ hydrofluoric acid or silane alone. In conclusion, this new study showed that the plasma treatment on the epoxy resin-based fiber post by the chair-side of the dental clinics is expected to be encouraging for the improved bond strength between an epoxy resin-based fiber post and core resin. This technology will have the potential to improve the endodontic restoration's success rate.

\subsection{Differentiation of Mesenchymal Stem Cells}

Human mesenchymal stem cells (hMSCs) are well-known to have the capability to differentiate into dental cells such as chondrocytes, osteoblasts, and adipocytes; however, the regulation of cellular differentiation, which will be crucial to successful dental regenerative medicine and tissue engineering, remains to be resolved in dental science. Kang et al. investigated the effects of plasma on the differentiation of hMSCs for the potential applications in tissue engineering and regenerative medicine [6]. They showed the differentiation of human mesenchymal stem cells using air, nitrogen gas and nonthermal biocompatible plasma. Their results indicated differential levels of cellular differentiation under different plasma exposure conditions. Their results may be due to the reactive oxygen species produced and the extracellular chemical environment induced by the plasma. Future efforts 
will be important to correlate differential differentiation using different parameters at the time of plasma treatments.

\subsection{Neuronal Regeneration}

The application of plasma also has the potential for facilitating central nervous system repair, which involves a complex interplay of different cells in the affected microenvironment, such as neurons and astrocytes. To investigate this, plasma was applied to neurons and astrocytes isolated from Sprague Dawley rat pups in order to stimulate injury recovery following a scratch-trauma assay [7]. Both cell types were treated with plasma and either cultured alone or together in a transwell system. Hormetic responses were observed after a direct plasma treatment, and a low dose plasma treatment resulted in a higher level of astrocyte regrowth. Interestingly, when astrocytes were treated with NTP and then co-cultured with untreated neurons, the neurite outgrowth was also increased. The results from this study suggest that plasma may have utility for neuronal injury repair and regeneration by affecting the microenvironment and further demonstrate the importance of optimizing stimulatory plasma treatment regimes.

\subsection{Immune Activation}

In the work "Plasma-Derived Reactive Species Shape a Differentiation Profile in Human Monocytes", a human cell line as well as primary human monocytes were investigated for their response to plasma treatment in vitro [8]. It was hypothesized that the plasmagenerated ROS/RNS contributes to differentiation-like behavior in these myeloid cells. Indeed, the surface marker treatment had changed in plasma-treated monocytes with regard to differentiation-associated molecules, and similar findings were made for the secretory cytokine and chemokine profiles, especially when analyzing mitogen-primed cells. Besides those functional consequences, plasma-treated monocytes showed greater tumor-toxic behavior than non-treated monocytes, suggesting that a toxicity and/or differentiation program could be initiated using the low-dose ROS/RNS treatment of these myeloid cells.

\subsection{Bio-Sterilization and Decontamination}

Nonthermal biocompatible plasma is a promising technology for the control of environmental pollution. Plasma-generated reactive species can be used to degrade organic and inorganic pollutants. Azadeh et al. reviewed various investigations into the use of nonthermal atmospheric pressure plasma technology to control organic wastewater purification. This study provided key outlines of the operational conditions, standards, parameters, and boundaries, and analyzed the continuing advancements related to the use of cold plasma in wastewater pollution control [9]. This review summarized previous investigations into the applications of plasma in water purification. Plasma production methods and their effectiveness have been investigated extensively. These investigations suggested that the results of interactions between the plasma and waste liquid could be utilized as an innovative water management technology that could eradicate pollutants in drinking water. Moreover, it is suggested that more thorough investigations with complex analytical methods are needed to elucidate the mechanisms implicated in plasma interactions with hazardous substances in water.

Several fungal species that release high amounts of pathogenic spores into the air are indoor air and food contaminants. Plasma can also be used to control fungal spores efficiently. A study by Noh et al. elucidated the death mechanism of fungal spores using a nonthermal biocompatible air gas plasma jet, simply called soft plasma jet [10]. They investigated the effect of plasma on the viability of spores and indicated that changes in cell wall rigidity and an induction of DNA damage were mainly affected by plasmatreated C. pruinosa spores. This study elucidated the effects of plasma-induced reactive species as the main stress source for fungal spores. Thus, plasma devices with a bio- 
sterilization function could be used for sanitary purposes in the medical manufacturing and food industries.

Funding: This research received no external funding.

Acknowledgments: Authors are supported by National Research Foundation under the Ministry of Science and ICT (NRF-2016K1A4A3914113) of the Korean Government, European Social Fund (ESF/14-BM-A55-0006-18) and German Federal Ministry of Education and Research (03Z22DN11, 03Z22Di1, 03Z22D511, 16GW0344k, 03COV06A), The Flanders Research Foundation (12S9221N, G044420N) and a Research Grant from Kwangwoon University in 2021.

Conflicts of Interest: The authors declare no conflict of interest.

\section{References}

1. Mitra, S.; Bhartiya, P.; Kaushik, N.; Nhat Nguyen, L.; Wahab, R.; Bekeschus, S.; Choi, E.H.; Kaushik, N.K. Plasma-Treated Flammulina velutipes-Derived Extract Showed Anticancer Potential in Human Breast Cancer Cells. Appl. Sci. 2020, $10,8395$. [CrossRef]

2. Adhikari, M.; Adhikari, B.; Kaushik, N.; Lee, S.-J.; Kaushik, N.K.; Choi, E.H. Melanoma Growth Analysis in Blood Serum and Tissue Using Xenograft Model with Response to Cold Atmospheric Plasma Activated Medium. Appl. Sci. 2019, 9, 4227. [CrossRef]

3. Bekeschus, S.; Moritz, J.; Helfrich, I.; Boeckmann, L.; Weltmann, K.-D.; Emmert, S.; Metelmann, H.-R.; Stoffels, I.; von Woedtke, T. Ex Vivo Exposure of Human Melanoma Tissue to Cold Physical Plasma Elicits Apoptosis and Modulates Inflammation. Appl. Sci. 2020, 10, 1971. [CrossRef]

4. Berner, J.; Seebauer, C.; Sagwal, S.K.; Boeckmann, L.; Emmert, S.; Metelmann, H.-R.; Bekeschus, S. Medical Gas Plasma Treatment in Head and Neck Cancer-Challenges and Opportunities. Appl. Sci. 2020, 10, 1944. [CrossRef]

5. Kim, H.-S.; Yang, S.-Y.; Choi, E.H.; Kim, K.-M.; Kwon, J.-S. Adhesion between Epoxy Resin-Based Fiber Post and Dental Core Resin Improved by Non-Thermal Atmospheric Pressure Plasma. Appl. Sci. 2020, 10, 2535. [CrossRef]

6. Kang, T.-Y.; Kwon, J.-S.; Kumar, N.; Choi, E.H.; Kim, K.-M. Effects of a Non-Thermal Atmospheric Pressure Plasma Jet with Different Gas Sources and Modes of Treatment on the Fate of Human Mesenchymal Stem Cells. Appl. Sci. 2019, 9, 4819. [CrossRef]

7. Katiyar, K.S.; Lin, A.; Fridman, A.; Keating, C.E.; Cullen, D.K.; Miller, V. Non-Thermal Plasma Accelerates Astrocyte Regrowth and Neurite Regeneration Following Physical Trauma In Vitro. Appl. Sci. 2019, 9, 3747. [CrossRef]

8. Freund, E.; Moritz, J.; Stope, M.; Seebauer, C.; Schmidt, A.; Bekeschus, S. Plasma-Derived Reactive Species Shape a Differentiation Profile in Human Monocytes. Appl. Sci. 2019, 9, 2530. [CrossRef]

9. Barjasteh, A.; Dehghani, Z.; Lamichhane, P.; Kaushik, N.; Choi, E.H.; Kaushik, N.K. Recent Progress in Applications of NonThermal Plasma for Water Purification, Bio-Sterilization, and Decontamination. Appl. Sci. 2021, 11, 3372. [CrossRef]

10. Noh, H.; Kim, J.E.; Kim, J.Y.; Kim, S.H.; Han, I.; Lim, J.S.; Ki, S.H.; Choi, E.H.; Lee, G.J. Spore Viability and Cell Wall Integrity of Cordyceps pruinosa Treated with an Electric Shock-Free, Atmospheric-Pressure Air Plasma Jet. Appl. Sci. 2019, 9, 3921. [CrossRef] 